\title{
A Note on Some Integrals by Malmsten and Bierens de Haan
}

\author{
Robert Reynolds*, Allan Stauffer \\ Department of Mathematics and Statistics, York University, Faculty of Science, Canada
}

Cite This Paper in the following Citation Styles

(a): [1] Robert Reynolds, Allan Stauffer, "A Note on Some Integrals by Malmsten and Bierens de Haan," Mathematics and Statistics, Vol.9, No.5, pp. 816-824, 2021. DOI: 10.13189/ms.2021.090522

(b): Robert Reynolds, Allan Stauffer, (2021). A Note on Some Integrals by Malmsten and Bierens de Haan. Mathematics and Statistics, 9(5), 816-824. DOI: 10.13189/ms.2021.090522

Copyright $\odot 2021$ by authors, all rights reserved. Authors agree that this article remains permanently open access under the terms of the Creative Commons Attribution License 4.0 International License

Abstract Carl Johan Malmsten (1846) and David Beirens de Haan (1847) published work containing some interesting integrals. While no formal derivations of the integrals in his book Nouvelles Tables d'Intégrales Défines are available in current literature deriving and evaluating such formulae are useful in all aspects of science and engineering whenever such formulae are used. Formulae in the book of Bierens de Haan are used in connection with certain potential problems where there is the need to determine the vector potential of two parallel, infinitely long, tubular rectangular conductors carrying cur-rents in opposite directions. In this current work we supply formal derivations for some of these integrals along with deriving some special cases as new integrals in order to expand upon the book of Bierens de haan to aid in potential research where these formulae are applicable. Updating book of integrals is always a useful exercise as it keeps the volume accurate and more useful for potential readers and researchers. Formal derivations are also useful as they help in verifying the correctness of integrals in such volumes. The definite integral we derived in this work is given by

$$
\int_{0}^{1} \frac{x^{-1-i m}\left(x^{2 i m}(\log (a)+i \log (x))^{k}+(\log (a)-i \log (x))^{k}\right)}{\left(-2 \cos (\lambda)+x^{-p}+x^{p}\right)} d x
$$

in terms of the Lerch function, where the parameters $a, k, m, \lambda$ and $p$ are general complex numbers subject to their restrictions. This formal derivation is then used to derive the correct version of a definite integral transform along with new formulae. Some of the results in this work are new.

Keywords Entries of Malmsten and Bierens de Haan, Logarithmic Integrals, Lerch Function

\section{Introduction}

In connection with the log log-integrals published by Malmsten [6] and definite integrals published by Bierens de Haan [5], the authors have the opportunity to evaluate integrals of the form

$$
\int_{0}^{1} \frac{x^{-1-i m}\left(x^{2 i m}(\log (a)+i \log (x))^{k}+(\log (a)-i \log (x))^{k}\right)}{\left(-2 \cos (\lambda)+x^{-p}+x^{p}\right)} d x
$$

in terms of the Lerch function, where the parameters $a, k, m, \lambda$ and $p$ are general complex numbers subject to their restrictions. This aim of this manuscript is to supply formal derivations for interesting integrals and also represent some integrals in terms of the Lerch function. By representing integrals in terms of the Lerch function, we widen the range of evaluation through analytic continuity, which in some cases was not present when derived by Malmsten [6] and Bierens de haan [5]. We also derive errata 
for one of the integrals in Malmsten [6] work which is always useful since his work is widely read. Regarding Entry 81 of [6], a closed formula is given which was also derived by Malmsten [6]. We also supply a table of integrals which makes these formulae easier to read. The derivations follow the method used by us in [8]. This method involves using a form of the generalized Cauchy's integral formula given by

$$
\frac{y^{k}}{k !}=\frac{1}{2 \pi i} \int_{C} \frac{e^{w y}}{w^{k+1}} d w .
$$

where $C$ is in general an open contour in the complex plane where the bilinear concomitant [8] has the same value at the end points of the contour. This method involves a form of equation (3) and multiplies both sides by a function, and take a definite integral of both sides. This yields a definite integral in terms of a contour integral. Then we multiply both sides of equation (3) by another function and take the infinite sum of both sides such that the contour integral of both equations are the same.

\section{Definite integral of the contour integral}

Using a generalization of Cauchy's integral formula we form two equations by replacing $y$ by $i x+\log (a)$ and multiplying by $e^{m x i}$ to form the first equation followed by replacing $x$ by $1 / x$ and multiplying by $e^{-i m x}$ to obtain the second, subtracting the two followed by multiplying by $\frac{1}{x\left(-2 \cos (\lambda)+x^{-p}+x^{p}\right)}$ then taking the definite integral with respect $x \in[0,1]$ to get

$$
\begin{aligned}
& \int_{0}^{1} \frac{x^{-1-i m}\left(x^{2 i m}(\log (a)+i \log (x))^{k}+(\log (a)-i \log (x))^{k}\right)}{2 k !\left(-2 \cos (\lambda)+x^{-p}+x^{p}\right)} d x \\
& =\frac{1}{2 \pi i} \int_{0}^{1} \int_{C} \frac{a^{w} w^{-k-1} \cos ((m+w) \log (x))}{x\left(-2 \cos (\lambda)+x^{-p}+x^{p}\right)} d w d x \\
& =\frac{1}{2 \pi i} \int_{C} \int_{0}^{1} \frac{a^{w} w^{-k-1} \cos ((m+w) \log (x))}{x\left(-2 \cos (\lambda)+x^{-p}+x^{p}\right)} d x d w \\
& =\frac{1}{2 \pi i} \int_{C} \frac{\pi a^{w} w^{-k-1} \csc (\lambda) \operatorname{csch}\left(\frac{\pi(m+w)}{p}\right) \sinh \left(\frac{(\pi-\lambda)(m+w)}{p}\right)}{2 p} d w
\end{aligned}
$$

from equation (404)(9) in [5] where $\operatorname{Re}(\lambda)<\pi$ and $-1<\operatorname{Re}(m+w)<0$. The cut is in the second $\mathrm{m}+\mathrm{w}$ quadrant starting at the origin and going vertically to infinity and the contour is on opposite sides of the cut going round the origin with zero radius. The logarithmic function is given for example in section (4.1) in [3]. We are able to switch the order of integration over $w+m$ and $x$ using Fubini's theorem since the integrand is of bounded measure over the space $\mathbb{C} \times[0,1]$. The result quoted is in error.

\section{The Lerch function and the Infinite sum of the contour integral}

The Lerch function is defined in section (2.2) in [9].

\subsection{Infinite sum of the contour integral}

In this section we will again use the generalized Cauchy's integral formula to derive equivalent contour integrals. First we replace $y$ by $y+t$ and multiply by $e^{m t}$ for the first equation and $y$ by $y-t$ and multiply by $e^{-m t}$ for second then subtract these two equations to get

$$
\frac{e^{m t}(t+y)^{k}-e^{-m t}(y-t)^{k}}{k !}=\frac{1}{2 \pi i} \int_{C} 2 w^{-k-1} e^{w y} \sinh (t(m+w)) d w
$$

Next we replace $t$ by $\frac{\pi-\lambda}{p}$ and multiply by $1 / 2$ to get

$$
\frac{e^{\frac{(\pi-\lambda) m}{p}}\left(\frac{\pi-\lambda}{p}+y\right)^{k}-e^{-\frac{(\pi-\lambda) m}{p}}\left(y-\frac{\pi-\lambda}{p}\right)^{k}}{2 k !}=\frac{1}{2 \pi i} \int_{C} w^{-k-1} e^{w y} \sinh \left(\frac{(\pi-\lambda)(m+w)}{p}\right) d w
$$

Next we replace $y$ by $\log (a)+\pi u(2 y+1)$ to get

$$
\begin{aligned}
& \frac{e^{\frac{(\pi-\lambda) m}{p}}\left(\log (a)+\frac{\pi-\lambda}{p}+\pi u(2 y+1)\right)^{k}-e^{-\frac{(\pi-\lambda) m}{p}}\left(\log (a)-\frac{\pi-\lambda}{p}+\pi u(2 y+1)\right)^{k}}{2 k !} \\
& =\frac{1}{2 \pi i} \int_{C} w^{-k-1} e^{w(\log (a)+\pi u(2 y+1))} \sinh \left(\frac{(\pi-\lambda)(m+w)}{p}\right) d w
\end{aligned}
$$


Next we multiply both sides by $e^{\pi m u(2 y+1)}$ to get

$$
\begin{gathered}
\frac{e^{\pi m u(2 y+1)}\left(e^{\frac{(\pi-\lambda) m}{p}}\left(\log (a)+\frac{\pi-\lambda}{p}+\pi u(2 y+1)\right)^{k}-e^{-\frac{(\pi-\lambda) m}{p}}\left(\log (a)-\frac{\pi-\lambda}{p}+\pi u(2 y+1)\right)^{k}\right)}{2 k !} \\
=\frac{1}{2 \pi i} \int_{C} w^{-k-1} \sinh \left(\frac{(\pi-\lambda)(m+w)}{p}\right) \exp (w(\log (a)+\pi u(2 y+1))+\pi m u(2 y+1)) d w
\end{gathered}
$$

Next we take the infinite sum over $y \in[0, \infty)$ simplify in terms of the Lerch function to get

$$
\begin{aligned}
& \frac{2^{k-1} \pi^{k} u^{k} e^{\frac{m(\lambda+\pi(p u-1))}{p}}\left(e^{\frac{2(\pi-\lambda) m}{p}} \Phi\left(e^{2 m \pi u},-k, \frac{p \pi u-\lambda+p \log (a)+\pi}{2 p \pi u}\right)-\Phi\left(e^{2 m \pi u},-k, \frac{p \pi u+\lambda+p \log (a)-\pi}{2 p \pi u}\right)\right)}{k !} \\
& =\frac{1}{2 \pi i} \sum_{y=0}^{\infty} \int_{C} w^{-k-1} \sinh \left(\frac{(\pi-\lambda)(m+w)}{p}\right) \exp (w(\log (a)+\pi u(2 y+1))+\pi m u(2 y+1)) d w \\
& =\frac{1}{2 \pi i} \int_{C} \sum_{y=0}^{\infty} w^{-k-1} \sinh \left(\frac{(\pi-\lambda)(m+w)}{p}\right) \exp (w(\log (a)+\pi u(2 y+1))+\pi m u(2 y+1)) d w \\
& =-\frac{1}{2 \pi i} \int_{C} \frac{1}{2} a^{w} w^{-k-1} \operatorname{csch}(\pi u(m+w)) \sinh \left(\frac{(\pi-\lambda)(m+w)}{p}\right) d w
\end{aligned}
$$

from equation (1.232.3) in [7]. Next we multiply both sides by $-\frac{2 \pi \csc (\lambda)}{p}$ and replace $u$ by $1 / p$ simplify to get

$$
\begin{aligned}
& -\frac{2^{k} \pi^{k+1}\left(\frac{1}{p}\right)^{k+1} \csc (\lambda) e^{\frac{\lambda m}{p}}\left(e^{\frac{2(\pi-\lambda) m}{p}} \Phi\left(e^{\frac{2 m \pi}{p}},-k, \frac{-\lambda+p \log (a)+2 \pi}{2 \pi}\right)-\Phi\left(e^{\frac{2 m \pi}{p}},-k, \frac{\lambda+p \log (a)}{2 \pi}\right)\right)}{k !} \\
& \quad=\frac{1}{2 \pi i} \int_{C} \frac{\pi a^{w} w^{-k-1} \csc (\lambda) \operatorname{csch}\left(\frac{\pi(m+w)}{p}\right) \sinh \left(\frac{(\pi-\lambda)(m+w)}{p}\right)}{p} d w
\end{aligned}
$$

from equation (1.232.3) in [7] given by

$$
\operatorname{csch}(x)=2 \sum_{k=0}^{\infty} e^{-(2 k+1) x}
$$

where $x>0$ and $\operatorname{csch}(i x)=-i \csc (x)$ from (4.5.10) in [3] and $\operatorname{Im}(m+w)>0$ in order for the sum to converge.

\section{Main Results}

\subsection{Definite integral in terms of the Lerch function}

Theorem 1. For all $a, k, m \in \mathbb{C}, \operatorname{Re}(\lambda)<\pi$,

$$
\begin{aligned}
& \int_{0}^{1} \frac{x^{-1-i m}\left(x^{2 i m}(\log (a)+i \log (x))^{k}+(\log (a)-i \log (x))^{k}\right)}{2\left(-2 \cos (\lambda)+x^{-p}+x^{p}\right)} d x \\
& =-2^{k-1} \pi^{k+1}\left(\frac{1}{p}\right)^{k+1} \csc (\lambda) e^{\frac{\lambda m}{p}}\left(e^{\frac{2(\pi-\lambda) m}{p}} \Phi\left(e^{\frac{2 m \pi}{p}},-k, \frac{-\lambda+p \log (a)+2 \pi}{2 \pi}\right)\right. \\
& \left.-\Phi\left(e^{\frac{2 m \pi}{p}},-k, \frac{\lambda+p \log (a)}{2 \pi}\right)\right)
\end{aligned}
$$

Proof. Since the right-hand sides of equations (4) and (10) are equal we may equate the left-hand sides simplifying the factorials to get the stated result. 


\subsection{Table of definite integrals}

In this section we will look at two definite integrals which are derived as special cases from equation (12). Starting by deriving two equations by setting $a=1$ and replacing $m$ by $-m$, followed by taking the difference of these two equations. In a similar manner we derive two other equations by adding the above equations. After deriving these two sets of equations we add them and simplify. We will replace $m$ by $i m$ and then again replace $m$ by $m-1$ to get

Theorem 2. For all $a, k, m \in \mathbb{C}, \operatorname{Re}(\lambda)<\pi$,

$$
\begin{array}{r}
\int_{0}^{1} \frac{x^{m} \log ^{k}\left(\frac{1}{x}\right)}{-2 \cos (\lambda)+x^{-p}+x^{p}} d x=-i 2^{k-1} \pi^{k+1}\left(\frac{1}{p}\right)^{k+1} \csc (\pi k) \csc (\lambda) e^{-\frac{1}{2} i \pi k-\frac{i \lambda(-m-1)}{p}}\left((-1)^{k} \Phi\left(e^{-\frac{2 i(-m-1) \pi}{p}},-k, \frac{\lambda}{2 \pi}\right)\right. \\
+(-1)^{k+1} e^{\frac{2 i(\lambda-\pi)(-m-1)}{p}} \Phi\left(e^{-\frac{2 i(-m-1) \pi}{p}},-k, 1-\frac{\lambda}{2 \pi}\right) \\
\left.-e^{\frac{2 i \lambda(-m-1)}{p}} \Phi\left(e^{\frac{2 i(-m-1) \pi}{p}},-k, \frac{\lambda}{2 \pi}\right)+e^{\frac{2 i \pi(-m-1)}{p}} \Phi\left(e^{\frac{2 i(-m-1) \pi}{p}},-k, 1-\frac{\lambda}{2 \pi}\right)\right)
\end{array}
$$

and when we replace $\lambda$ by $\lambda+\pi$ we get the second equation

Theorem 3. For all $a, k, m, \lambda \in \mathbb{C}$,

$$
\begin{aligned}
& \int_{0}^{1} \frac{x^{m} \log ^{k}\left(\frac{1}{x}\right)}{2 \cos (\lambda)+x^{-p}+x^{p}} d x=i 2^{k-1} \pi^{k+1}\left(\frac{1}{p}\right)^{k+1} \csc (\pi k) \csc (\lambda) e^{-\frac{1}{2} i \pi k-\frac{i(\lambda+\pi)(-m-1)}{p}}\left(( - 1 ) ^ { k } \Phi \left(e^{-\frac{2 i(-m-1) \pi}{p}}\right.\right. \\
& \left.-k, \frac{\lambda+\pi}{2 \pi}\right)+(-1)^{k+1} e^{\frac{2 i \lambda(-m-1)}{p}} \Phi\left(e^{-\frac{2 i(-m-1) \pi}{p}},-k, 1-\frac{\lambda+\pi}{2 \pi}\right) \\
& \left.-e^{\frac{2 i(\lambda+\pi)(-m-1)}{p}} \Phi\left(e^{\frac{2 i(-m-1) \pi}{p}},-k, \frac{\lambda+\pi}{2 \pi}\right)+e^{\frac{2 i \pi(-m-1)}{p}} \Phi\left(e^{\frac{2 i(-m-1) \pi}{p}},-k, 1-\frac{\lambda+\pi}{2 \pi}\right)\right)
\end{aligned}
$$

We will use these two theorems to derive the following table of integrals. We will also use the following equations to assist in simplification of the Lerch function for the given special cases.

Entry (4) in Table below (64:12:7) in [2]

$$
\Phi(1, v, 2 u)=\frac{\zeta(v, u)+\zeta(v, u+1 / 2)}{2}
$$

Equation (64:13:3) in [2]

$$
\eta(v, 2 u)=2^{-v}(\zeta(v, u)-\zeta(v, u+1 / 2))
$$

The Polylogarithm funcion is given by equation (1.11.14) in [4]

$$
\begin{aligned}
F(z, s) & =\sum_{n=1}^{\infty}\left(z^{n} / n^{s}\right) \\
& =z \Phi(z, s, 1)
\end{aligned}
$$

\subsubsection{Derivation of entry 81 in [6]}

Using equation (14) we set $p=1$. Then we form two equations by replacing $m$ by $m-3 / 2$ for the first equation and $m-1 / 2$ for the second equation. We then add these two equations to get

$$
\begin{aligned}
& \int_{0}^{1} \frac{(x+1) x^{m-\frac{1}{2}} \log ^{k}\left(\frac{1}{x}\right)}{x^{2}+2 x \cos (\lambda)+1} d x=2^{k-1}(-1 \\
& \left.+e^{i \lambda}\right) \pi^{k+1} \csc (\pi k) \csc (\lambda) e^{-\frac{1}{2} i(\pi k+\lambda+2(\lambda+\pi) m)}\left(e^{2 i \lambda m} \Phi\left(-e^{-2 i m \pi},-k, \frac{\pi-\lambda}{2 \pi}\right)\right. \\
& +\Phi\left(-e^{-2 i m \pi},-k, \frac{\lambda+\pi}{2 \pi}\right) \\
& \left.-e^{i \pi(k+2 m)}\left(\Phi\left(-e^{2 i m \pi},-k, \frac{\pi-\lambda}{2 \pi}\right)+e^{2 i \lambda m} \Phi\left(-e^{2 i m \pi},-k, \frac{\lambda+\pi}{2 \pi}\right)\right)\right)
\end{aligned}
$$




\subsubsection{Derivation of entry 4.271 .13 in [7]}

Using equation (13) we will derive two equations by first setting $m=-1, p=-1$ and replacing $\lambda$ by $2 a \pi$ and then for the second equation again using equation (13) and setting $m=0, p=1$ and replacing $\lambda$ by $2 a \pi$ and taking the difference, simplifying in terms of the Hurwitz zeta function $\zeta(v, u)$ to get

$$
\int_{0}^{1} \frac{(\cos (2 \pi a)-x) \log ^{2 k+1}\left(\frac{1}{x}\right)}{-2 x \cos (2 \pi a)+x^{2}+1} d x=-4^{k} \pi^{2 k+2} \sec (\pi k)(\zeta(-2 k-1,1-a)+\zeta(-2 k-1, a))
$$

from equations (25.14.2) in [1] and (64:13:3) in [2].

\subsubsection{Derivation of entry 4.272 .1 in [7]}

Using equation (14) and setting $m=-1, p=1$ and replacing $k$ by $q-1$ and $\lambda$ by $t$ simplify to get

$$
\int_{0}^{1} \frac{\log ^{q-1}\left(\frac{1}{x}\right)}{2 x \cos (t)+x^{2}+1} d x=2^{q-2} e^{-\frac{1}{2} i \pi q}\left(1+e^{i \pi q}\right) \pi^{q} \csc (\pi q) \csc (t)\left(\zeta\left(1-q, \frac{\pi-t}{2 \pi}\right)-\zeta\left(1-q, \frac{t+\pi}{2 \pi}\right)\right)
$$

from equations (25.14.2) in [1] and (64:13:3) in [2].

\subsubsection{Derivation of entry 4.272 .2 in [7]}

Using equation (14) and setting $m=0, p=1$ and replacing $k$ by $q-1$ and $\lambda$ by $t$ simplify to get

$$
\begin{aligned}
\int_{0}^{1} \frac{(x-1) \log ^{q-1}\left(\frac{1}{x}\right)}{2 x \cos (t)+x^{2}+1} d x=2^{q-1} \pi^{q} \csc (\pi q) \csc \left(\frac{t}{2}\right) & \left(\zeta\left(1-q, \frac{t+\pi}{2 \pi}\right) \cos \left(\frac{1}{2}(\pi q+t)\right)\right. \\
& \left.-\zeta\left(1-q, \frac{\pi-t}{2 \pi}\right) \sin \left(\frac{1}{2}(-\pi q+t+\pi)\right)\right)
\end{aligned}
$$

from equation (25.14.2) in [1] and entry (4) in Table below (64:12:7) in [2].

\subsubsection{Derivation of entry 63 in [6]}

Using equation (14) we first set $m=-1, p=1$. Then we take the first partial derivative with respect to $k$ followed by applying L'Hopital's rule as $k \rightarrow 0$ to the right-hand side simplify to get

$$
\int_{0}^{1} \frac{\log \left(\log \left(\frac{1}{x}\right)\right)}{x^{2}+2 x \cos (\lambda)+1} d x=\frac{1}{2} \csc (\lambda)\left(\lambda \log (2 \pi)+\pi \log \left(\frac{(\lambda-\pi) \Gamma\left(\frac{1}{2}\left(\frac{\lambda}{\pi}-1\right)\right)}{(-\lambda-\pi) \Gamma\left(-\frac{\lambda+\pi}{2 \pi}\right)}\right)\right)
$$

from equations (9.533.3) in [7], (25.14.2) in [1] and (64:13:3) in [2].

\subsubsection{Derivation of entry 58 in [6]}

In this derivation we will form two equations. For the first equation we use equation (14) we set $p=1$ and replace $m$ by $m-1$. To form the second equation we simply apply L'Hopital's rule to the right-hand side and simplify. Then we multiply the first equation by $\sin (\lambda)$ and take the differenec of the two simplify to get

$$
\begin{aligned}
\int_{0}^{1} x^{m} \log ^{s-1}\left(\frac{1}{x}\right)\left(\frac{\sin (\lambda)}{x^{2}+2 x \cos (\lambda)+1}-\frac{1}{(x+1)^{2}}\right) d x \\
=-i 2^{s-2} \pi^{s} \csc (\pi s)\left(i e ^ { - \frac { 1 } { 2 } i ( 2 ( \lambda + \pi ) m + \pi s ) } \left(e^{2 i \lambda m} \Phi\left(e^{-2 i m \pi}, 1-s, \frac{\pi-\lambda}{2 \pi}\right)-\Phi\left(e^{-2 i m \pi}, 1-s, \frac{\lambda+\pi}{2 \pi}\right)\right.\right. \\
\left.+e^{i \pi(2 m+s)}\left(\Phi\left(e^{2 i m \pi}, 1-s, \frac{\pi-\lambda}{2 \pi}\right)-e^{2 i \lambda m} \Phi\left(e^{2 i m \pi}, 1-s, \frac{\lambda+\pi}{2 \pi}\right)\right)\right) \\
+\frac{e^{-\frac{1}{2} i \pi(2 m+s-1)}}{\pi}\left(-2 i \pi m \Phi\left(e^{-2 i m \pi}, 1-s, \frac{1}{2}\right)+(s-1) \Phi\left(e^{-2 i m \pi}, 2-s, \frac{1}{2}\right)\right. \\
\left.\left.+e^{i \pi(2 m+s)}\left(2 i \pi m \Phi\left(e^{2 i m \pi}, 1-s, \frac{1}{2}\right)+(s-1) \Phi\left(e^{2 i m \pi}, 2-s, \frac{1}{2}\right)\right)\right)\right)
\end{aligned}
$$

This is the Lerch representation for entry 58 in [6]. 


\subsubsection{Derivation of entry 29 in [6]}

Using equation (14) setting $p=1$ and replacing $m$ by $m-1$ and $k$ by $s-1$ multiply both sides by $\frac{\sin (\lambda)}{\Gamma(s)} \operatorname{simplify}$ we get

$$
\begin{aligned}
& \frac{\sin (\lambda)}{\Gamma(s)} \int_{0}^{1} \frac{x^{m} \log ^{s-1}\left(\frac{1}{x}\right)}{\left(x^{2}+2 x \cos (\lambda)+1\right)} d x=\frac{2^{s-2} \pi^{s}}{\Gamma(s)} \csc (\pi s) e^{-\frac{1}{2} i(2(\lambda+\pi) m+\pi s)}\left(e^{2 i \lambda m} \Phi\left(e^{-2 i m \pi}, 1-s, \frac{\pi-\lambda}{2 \pi}\right)\right. \\
& -\Phi\left(e^{-2 i m \pi}, 1-s, \frac{\lambda+\pi}{2 \pi}\right) \\
& \left.+e^{i \pi(2 m+s)}\left(\Phi\left(e^{2 i m \pi}, 1-s, \frac{\pi-\lambda}{2 \pi}\right)-e^{2 i \lambda m} \Phi\left(e^{2 i m \pi}, 1-s, \frac{\lambda+\pi}{2 \pi}\right)\right)\right)
\end{aligned}
$$

\subsubsection{Derivation of entry 30 in [6]}

Using equation (14) setting $p=1$ and replacing $m$ by $m-1$ and $k$ by $s-1$ multiply both sides by $\frac{\sin (\lambda) \sec \left(\frac{\pi s}{2}\right)}{\Gamma(s)} \operatorname{simplify}$ we get

$$
\begin{aligned}
& \frac{\sin (\lambda) \sec \left(\frac{\pi s}{2}\right)}{\Gamma(s)} \int_{0}^{1} \frac{x^{m} \log ^{s-1}\left(\frac{1}{x}\right)}{\left(x^{2}+2 x \cos (\lambda)+1\right)} d x=\frac{2^{s-2} \pi^{s}}{\Gamma(s)} \csc (\pi s) \sec \left(\frac{\pi s}{2}\right) e^{-\frac{1}{2} i(2(\lambda+\pi) m+\pi s)}\left(e ^ { 2 i \lambda m } \Phi \left(e^{-2 i m \pi}, 1\right.\right. \\
& \left.-s, \frac{\pi-\lambda}{2 \pi}\right)-\Phi\left(e^{-2 i m \pi}, 1-s, \frac{\lambda+\pi}{2 \pi}\right) \\
& \left.+e^{i \pi(2 m+s)}\left(\Phi\left(e^{2 i m \pi}, 1-s, \frac{\pi-\lambda}{2 \pi}\right)-e^{2 i \lambda m} \Phi\left(e^{2 i m \pi}, 1-s, \frac{\lambda+\pi}{2 \pi}\right)\right)\right)
\end{aligned}
$$

\subsubsection{Derivation of entry 31 in [6]}

Using equation (14) setting $p=1, m=-1$ and replacing $k$ by $s-1$ and $\lambda$ by $\frac{m \pi}{n}$ multiply both sides by $\frac{\pi^{1-s}}{\Gamma(s)}\left(\frac{1}{n}\right)^{1-s} \sec \left(\frac{\pi s}{2}\right) \sin \left(\frac{\pi m}{n}\right)$ simplify we get

$$
\begin{aligned}
& \int_{0}^{1} \frac{\pi^{1-s}\left(\frac{1}{n}\right)^{1-s} \sec \left(\frac{\pi s}{2}\right) \sin \left(\frac{\pi m}{n}\right) \log ^{s-1}\left(\frac{1}{x}\right)}{\Gamma(s)\left(2 x \cos \left(\frac{\pi m}{n}\right)+x^{2}+1\right)} d x=\frac{\pi 2^{s-2}}{\Gamma(s)} e^{-\frac{1}{2} i \pi s}\left(1+e^{i \pi s}\right)\left(\frac{1}{n}\right)^{1-s} \csc (\pi s) \sec \left(\frac{\pi s}{2}\right)\left(\zeta\left(1-s, \frac{n-m}{2 n}\right)\right. \\
& \left.-\zeta\left(1-s, \frac{m+n}{2 n}\right)\right)
\end{aligned}
$$

from equations (25.14.2) in [1] and (64:13:3) in [2].

\subsubsection{Derivation of entry 32 in [6]}

Using equation (14) setting $p=1, m=-1$ and replacing $k$ by $s-1$ and $\lambda$ by $\frac{\pi}{n}$ multiply both sides by $\frac{\pi^{1-s}\left(\frac{1}{n}\right)^{1-s} \sec \left(\frac{\pi s}{2}\right) \sin \left(\frac{\pi m}{n}\right)}{\Gamma(s)}$ simplify we get

$$
\begin{aligned}
& \int_{0}^{1} \frac{\pi^{1-s}\left(\frac{1}{n}\right)^{1-s} \sin \left(\frac{\pi}{n}\right) \sec \left(\frac{\pi s}{2}\right) \log ^{s-1}\left(\frac{1}{x}\right)}{\Gamma(s)\left(2 x \cos \left(\frac{\pi}{n}\right)+x^{2}+1\right)} d x=\frac{\pi 2^{s-2}}{\Gamma(s)} e^{-\frac{1}{2} i \pi s}\left(1+e^{i \pi s}\right)\left(\frac{1}{n}\right)^{1-s} \csc (\pi s) \sec \left(\frac{\pi s}{2}\right)\left(\zeta\left(1-s, \frac{n-1}{2 n}\right)\right. \\
& \left.-\zeta\left(1-s, \frac{n+1}{2 n}\right)\right)
\end{aligned}
$$




\subsubsection{Derivation of entry 33 in [6]}

Using equation (14) setting $p=1, m=-1, \lambda=\frac{\pi}{3}$ and replacing $k$ by $s-1$ and simplify we get

$$
\int_{0}^{1} \frac{\log ^{s-1}\left(\frac{1}{x}\right)}{x^{2}+x+1} d x=\frac{2^{s-1} \pi^{s}}{\sqrt{3}}\left(\zeta\left(1-s, \frac{1}{3}\right)-\zeta\left(1-s, \frac{2}{3}\right)\right) \csc \left(\frac{\pi s}{2}\right)
$$

and using equation (14) setting $p=1, m=-1, \lambda=\frac{\pi}{2}$ and replacing $k$ by $s-1$ and simplify we get

$$
\int_{0}^{1} \frac{\log ^{s-1}\left(\frac{1}{x}\right)}{x^{2}+1} d x=2^{s-2} e^{-\frac{1}{2} i \pi s}\left(1+e^{i \pi s}\right) \pi^{s}\left(\zeta\left(1-s, \frac{1}{4}\right)-\zeta\left(1-s, \frac{3}{4}\right)\right) \csc (\pi s)
$$

from equations (25.14.2) in [1] and (64:13:3) in [2].

\subsubsection{Derivation of entry 36 in [6]}

Using equation (14) setting $p=1, m=-1, \lambda=\frac{\pi}{3}$ and replacing $k$ by $s-1$ taking the first partial derivative with respect to $s$ and simplify we get

$$
\begin{aligned}
\int_{0}^{1} \frac{\log \left(\log \left(\frac{1}{x}\right)\right) \log ^{s-1}\left(\frac{1}{x}\right)}{x^{2}+x+1} d x=-\frac{2^{s-2} \pi^{s} \csc \left(\frac{\pi s}{2}\right)}{\sqrt{3}}\left(2 \zeta^{\prime}\left(1-s, \frac{1}{3}\right)-2 \zeta^{\prime}\left(1-s, \frac{2}{3}\right)\right. \\
\left.+\left(\zeta\left(1-s, \frac{1}{3}\right)-\zeta\left(1-s, \frac{2}{3}\right)\right)\left(\pi \cot \left(\frac{\pi s}{2}\right)+\log \left(\frac{1}{4 \pi^{2}}\right)\right)\right)
\end{aligned}
$$

and using equation (14) setting $p=1, m=-1, \lambda=\frac{\pi}{2}$ and replacing $k$ by $s-1$ taking the first partial derivative with respect to $s$ and simplify we get

$$
\begin{array}{r}
\int_{0}^{1} \frac{\log \left(\log \left(\frac{1}{x}\right)\right) \log ^{s-1}\left(\frac{1}{x}\right)}{x^{2}+1} d x=-2^{s-3} e^{-\frac{1}{2} i \pi s} \pi^{s}\left(\cot \left(\frac{\pi s}{2}\right)+i\right)\left(2\left(\zeta^{\prime}\left(1-s, \frac{1}{4}\right)-\zeta^{\prime}\left(1-s, \frac{3}{4}\right)\right)\right. \\
\left.+\left(\zeta\left(1-s, \frac{1}{4}\right)-\zeta\left(1-s, \frac{3}{4}\right)\right)\left(\pi \cot \left(\frac{\pi s}{2}\right)-2 \log (2 \pi)\right)\right)
\end{array}
$$

from equations (25.14.2) in [1] and (64:13:3) in [2].

\subsubsection{Derivation of entry 37 in [6]}

Using equation (14) setting $p=-1, \lambda=\pi / 3$ and $m=-1$ then taking the first partial derivative with respect to $k$ then setting $k=-1 / 2$ simplify we get

$\int_{0}^{1} \frac{\log \left(\log \left(\frac{1}{x}\right)\right)}{\left(x^{2}+x+1\right) \sqrt{\log \left(\frac{1}{x}\right)}} d x=\frac{1}{2} i \sqrt{\frac{\pi}{3}}\left(-2 \zeta^{\prime}\left(\frac{1}{2}, \frac{1}{3}\right)+2 \zeta^{\prime}\left(\frac{1}{2}, \frac{2}{3}\right)+\left(\zeta\left(\frac{1}{2}, \frac{1}{3}\right)-\zeta\left(\frac{1}{2}, \frac{2}{3}\right)\right)\left(\log \left(4 \pi^{2}\right)+(-1+2 i) \pi\right)\right)$

and using equation (14) setting $p=-1, \lambda=\pi / 2$ and $m=-1$ then taking the first partial derivative with respect to $k$ then setting $k=-1 / 2$ simplify we get

$$
\int_{0}^{1} \frac{\log \left(\log \left(\frac{1}{x}\right)\right)}{\left(x^{2}+1\right) \sqrt{\log \left(\frac{1}{x}\right)}} d x=\frac{1}{4} \sqrt{\pi}\left(\left(\zeta\left(\frac{1}{2}, \frac{3}{4}\right)-\zeta\left(\frac{1}{2}, \frac{1}{4}\right)\right)((2+i) \pi-2 i \log (2 \pi))-2 i\left(\zeta^{\prime}\left(\frac{1}{2}, \frac{1}{4}\right)-\zeta^{\prime}\left(\frac{1}{2}, \frac{3}{4}\right)\right)\right)
$$

from equations (25.14.2) in [1] and (64:13:3) in [2].

\subsubsection{Some special cases involving new definite integral forms}

Using equation (12) and setting $m=0$ and replacing $a$ by $e^{i \log (a)}$ simplify we get

$$
\begin{array}{r}
\int_{0}^{1} \frac{(\log (a)-\log (x))^{k}+(\log (a)+\log (x))^{k}}{x\left(-2 \cos (\lambda)+x^{-p}+x^{p}\right)} d x=-\left(\frac{i}{2}\right)^{-k} \pi^{k+1}\left(\frac{1}{p}\right)^{k+1} \csc (\lambda)\left(\zeta\left(-k, \frac{-\lambda+i p \log (a)+2 \pi}{2 \pi}\right)\right. \\
\left.-\zeta\left(-k, \frac{\lambda+i p \log (a)}{2 \pi}\right)\right)
\end{array}
$$




\subsection{Example 1}

Using equation (34) and applying L'Hopital's rule to the right-hand side as $k \rightarrow-1$ simplify we get

$$
\int_{0}^{1} \frac{x^{p-1}}{\left(\log ^{2}(a)-\log ^{2}(x)\right)\left(-2 \cos (\lambda) x^{p}+x^{2 p}+1\right)} d x=\frac{i \csc (\lambda)}{4 \log (a)}\left(\psi^{(0)}\left(\frac{-\lambda+i p \log (a)+2 \pi}{2 \pi}\right)-\psi^{(0)}\left(\frac{\lambda+i p \log (a)}{2 \pi}\right)\right)
$$

from equation (1.11.14) in [4].

\subsubsection{Derivation of Table 27 entry (22) in [5]}

Using equation (34) and setting $k=1$ simplify using entry (2) in Table below (64:4:2) in [2] we get

$$
\int_{0}^{1} \frac{d x}{x\left(x^{-p}-2 \cos (\lambda)+x^{p}\right)}=\frac{(\pi-\lambda) \csc (\lambda)}{2 p}
$$

\section{Discussion}

The work of Malmsten contains definite $\log \log$-integrals. The work by Bierens de Haan contains a vast number of definite integrals. We have been able to derive the correct versions of one these integrals or supply the analytically continued closed forms. These results will assist readers to have the up to date versions of the solutions to these integrals.

\section{Conclusion}

In this paper, we have presented a novel method for deriving some interesting definite integrals using contour integration. The results presented were numerically verified for both real and imaginary and complex values of the parameters in the integrals using Mathematica by Wolfram

\section{Acknowledgments}

This research is supported by NSERC Canada under Grant 504070

\section{Conflict of Interest}

The authors confirm there are no conflicts of interest.

\section{REFERENCES}

[1] Frank W.J. Olver, Daniel W. Lozier, Ronald F. Boisvert, and Charles W. Clark (eds.) NIST Digital Library of Mathematical Functions, U.S. Department of Commerce, National Institute of Standards and Technology, Washington, DC; Cambridge University Press, Cambridge, 2010, With 1 CD-ROM (Windows, Macintosh and UNIX). MR 2723248 (2012a:33001)

[2] Keith B Oldham; Jan C Myland; Jerome Spanier, An atlas of functions : with Equator, the atlas function calculator, New York, NY, Springer, 2009, English : 2nd ed, pp. 1-762.

[3] Abramowitz, M. and Stegun, I.A.(Eds), Handbook of Mathematical Functions with Formulas, Graphs, and Mathematical Tables, 9th printing, New York, Dover, (1982), pp. 1-1044.

[4] A. Erdéyli, W. Magnus, F. Oberhettinger, and F.G. Tricomi Higher Transcendental Functions Vol. I, 1953a, McGraw-Hill Book Company, Inc., New York-Toronto-London, pp. 1-325.

[5] Bierens de Haan, D., Nouvelles Tables d'intégrales définies, Leide, P.Engels, Libraire Éditeur, Amsterdam, 1867, pp. $1-762$.

[6] Malmsten, C.J., De integralibus quibusdam definitis seriebusque infinitis (Eng. trans.: On some definite integrals and series), J. Reine Angew. Math., 38, pp. 1-39, 1846. 
[7] Gradshteyn I.S \& Ryzhik I.M, Tables of Integrals, Series and Products, 6 Ed, Academic Press (2000), USA, pp. 1-1220.

[8] Reynolds, R.; Stauffer, A. A Method for Evaluating Definite Integrals in Terms of Special Functions with Examples. 2020, International Mathematical Forum, Vol. 15, 2020, no. 5, 235-244. https://doi.org/10.12988/imf.2020.91272

[9] Reynolds, R.; Stauffer, A. Simultaneous Contour Method of a Trigonometric Integral by Prudnikov et al., Mathematics 2021, 9, 1453. https://doi.org/10.3390/math9121453 